\title{
Production of pure amorphous silica from wheat straw ash
}

Vilém Bartůněk PhD

Department of Inorganic Chemistry, Faculty of Chemical Technology, University of Chemistry and Technology, Prague, Czech Republic (corresponding author: vilem.bartunek@vscht.cz)

\section{David Sedmidubský PhD}

Department of Inorganic Chemistry, Faculty of Chemical Technology, University of Chemistry and Technology, Prague, Czech Republic

\author{
Daniel Bouša MSC \\ Department of Inorganic Chemistry, Faculty of Chemical Technology, \\ University of Chemistry and Technology, Prague, Czech Republic \\ Ondřej Jankovský PhD \\ Department of Inorganic Chemistry, Faculty of Chemical Technology, \\ University of Chemistry and Technology, Prague, Czech Republic
}

The product of a local energy facility focused on biomass, wheat straw ash waste was processed to pure amorphous silica and other phases that can be further used by thermal treatment with sodium hydroxide and subsequent leaching and acid precipitation by sulfur dioxide $\left(\mathrm{SO}_{2}\right)$ or carbon dioxide $\left(\mathrm{CO}_{2}\right)$. Extra-pure amorphous silica can be prepared using the sulfur dioxide-based process; the more affordable carbon dioxide precipitation process may be used for applications where ultrapure amorphous silica is not required. The obtained products were characterized by X-ray powder diffraction, scanning electron microscopy, energy-dispersive spectroscopy and X-ray fluorescence. The method displays a potential to be scaled up and applied in industrial practice. In this way, not only is the valuable amorphous silica obtained, but also other phases resulting from the process can be optionally utilized. All processes are facile, environmentally friendly and oil independent, particularly when focused on the carbon dioxide route.

\section{Introduction}

Waste biomass is a promising renewable energy source. It is available in agricultural regions in large quantities; it is easily processed and stored which makes it suitable as a local bioenergy source. Also, biomass can be used for production of liquid, solid and gas fuel supplies, allowing its application according to the needs of local facilities. It follows from the above that in comparison with solar and wind technologies, biomass-based technologies have low dependence on weather and climatic imbalances. Also, the respective technologies used are established, well known and perform well.

Amorphous and fine silica particles with large surface area are used in many applications including adhesives, plastics, sealants, coatings, inks, toner, cosmetics, food additives and defoamers. Their use in catalysis $^{1-3}$ is also of high importance. More recently, amorphous silica has been examined, for example, for printing of cotton fabric, ${ }^{4}$ for controlled drug release, ${ }^{5,6}$ in the form of composite materials for flame retardancy, ${ }^{7}$ or in advanced composite materials. $^{8-11}$ Amorphous silica based on ash as a raw material was previously prepared by the hydrochloride acidification of fly ash with the assistance of cetyltrimethylammonium bromide and polyethylene glycol, ${ }^{12}$ from corn cob ash at variable $\mathrm{pH}$ conditions by means of sol-gel processing ${ }^{13}$ and by dissolution-precipitation from rice straw ash. ${ }^{14}$

Biomass ash is a problematic waste because it contains a relatively large fraction of silicon oxide, which is insoluble in most solvents. Hydrofluoric acid as a leaching agent is dangerous ${ }^{15}$ and environmentally incompatible, while leaching by solutions of hydroxides is time consuming and with limited efficiency in some cases. Therefore, the possibility of thermal processing by sodium hydroxide to silicates seems to be a useful and promising direction. ${ }^{16}$

Biomass ash often contains a low concentration of toxic metals such as lead, zinc, or cadmium, which limit the usage of the ash. If the biomass ash is pure, it can be used as a fertilizer. However, if it contains an elevated concentration of heavy metals, ${ }^{17}$ large volumes of waste are produced that have to be stored in landfills that are highly expensive. For example, the Zluticka heating plant in the Czech Republic burns approximately $4000 \mathrm{t}$ of wooden chips and $1000 \mathrm{t}$ of wheat straw producing tens of tons of biomass ash every year. Hence, it is of great importance to find a way how to utilize such ash. Also there is a necessity to address the economy of the proposed procedures with respect to the environmental impact in a wide perspective; therefore, affordable and environmentally safe substances have to be used.

In this study, amorphous silica was prepared by a modified acid precipitation method from wheat straw ash using only a few basic chemicals. Such a route can be simply upscaled, which will make it affordable for industrial use.

\section{Experimental}

Wheat straw ash was obtained from the Zluticka heating plant in the Czech Republic. First $2.0 \mathrm{~g}$ of ash was mixed with $3.0 \mathrm{~g}$ of sodium hydroxide (Lach-Ner, Czech Republic, p.a.) and homogenized. Next, the mixture was moved into a stainless steel container and thermally treated at $320^{\circ} \mathrm{C}$ for $1 \mathrm{~h}$. Next, the 
obtained fritted solid black powder carbon was removed by simply pouring it out, and the remaining solid was moved to water. The obtained suspension was filtered using a suction set-up and filtration paper. The filtration cake was divided by weight by rapid decantation to low and high mass fractions (discussed in the next paragraph). The filtrate was then subjected to acidification by carbon dioxide $\left(\mathrm{CO}_{2}\right)$ or by sulfur dioxide $\left(\mathrm{SO}_{2}\right)$. The solid obtained by centrifugation was washed to neutral $\mathrm{pH}$, acidified slightly by few drops of hydrochloride acid and next carefully washed by distilled water and dried. The whole procedure is schematically shown in Figure 1. Samples were termed 'ash,' 'silica- $\mathrm{CO}_{2}$ ' and 'silica- $\mathrm{SO}_{2}$ ' according to the synthesis procedure.

The separation of various fractions from the obtained solid will be described in the following paragraph. In this process, the solid was dispersed into water and stirred. After the stirring, the suspension was allowed to sediment for $20 \mathrm{~s}$. Subsequently, liquid containing the light phase was cast off. The remaining sediment was the heavy phase. The light phase was then filtered by suction filtration from the suspension and dried.

X-ray powder diffraction (XRD) data were collected at room temperature with a D2 Phaser powder diffractometer using cobalt (Co) $\mathrm{K}_{\alpha}$ radiation $(\lambda=1.7902 \AA)$. The X'Pert HighScore Plus program was used for data processing.

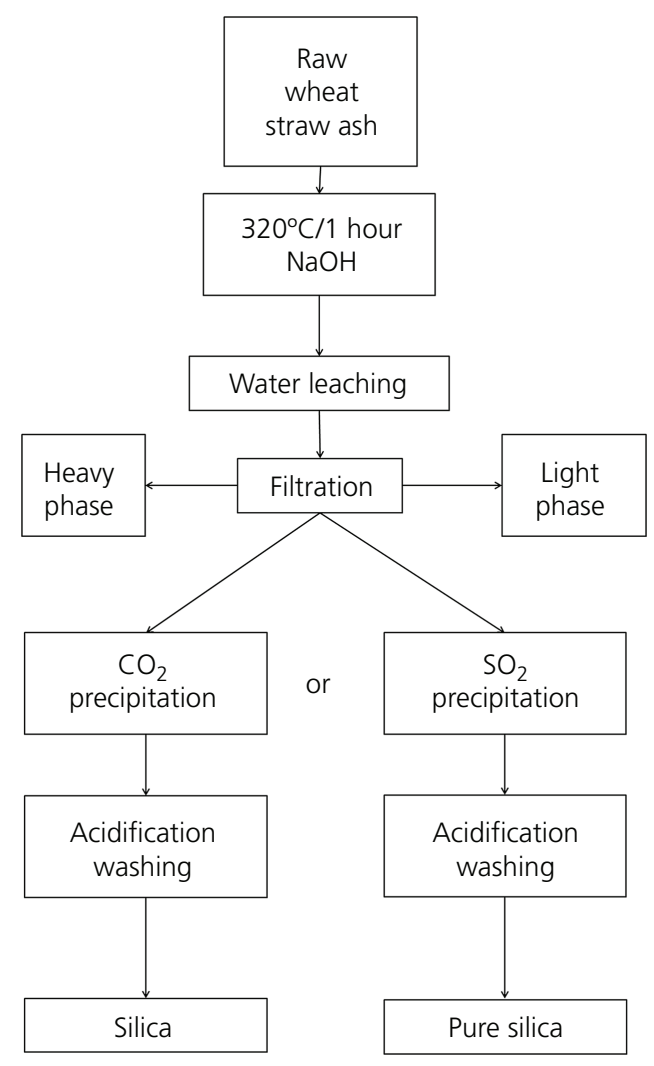

Figure 1. Schematic diagram of the experimental procedure
X-ray fluorescence analysis (XRF) was performed using an Axios sequential WD-XRF spectrometer (Panalytical, the Netherlands) equipped with a rhodium anode end-window X-ray tube fitted with a $50 \mu \mathrm{m}$ beryllium window. The resulting data were collected by SuperQ software and further evaluated by Omnian software. The analyzed powders were pressed, without any binding agent, onto boric acid $\left(\mathrm{H}_{3} \mathrm{BO}_{3}\right)$ pellets with a total thickness of approximately $5 \mathrm{~mm}$ and a diameter of $40 \mathrm{~mm}$. The pellets were then covered with a $4 \mu \mathrm{m}$ supporting polypropylene film.

The morphology was examined using scanning electron microscopy (SEM) with a field emission gun electron source (Tescan Lyra dual-beam microscope). Elemental composition and mapping were performed using an energy-dispersive spectroscopy (EDS) analyzer $\left(\mathrm{X}-\mathrm{Max}^{\mathrm{N}}\right.$ ) with a $20 \mathrm{~mm}^{2} \mathrm{SDD}$ detector (Oxford Instruments) and the AZtecEnergy software. To conduct the measurements, the samples were placed on a carbon conductive tape. SEM and EDS measurements were carried out using a $15 \mathrm{kV}$ electron beam.

\section{Results and discussion}

In the first step, the wheat straw ash was characterized in detail by XRD, SEM, EDS and XRF. The presence of a higher amount of carbon is obvious from the first view. XRD confirmed that the wheat straw ash is partially amorphous, and it contains two major phases. These phases are quartz (main reflection at $2 \theta=31 \cdot 2^{\circ}$ ) and cristobalite (main reflection at $2 \theta=25 \cdot 7^{\circ}$ ). The XRD patterns are shown in Figure 2. The amount of amorphous phase was determined as $85.3 \mathrm{wt} . \%$ from the Rietveld refinement. The composition of wheat straw ash was measured using XRF (results are shown in Table 1). High amounts of carbon, silicon and potassium were obtained. These results were confirmed by EDS

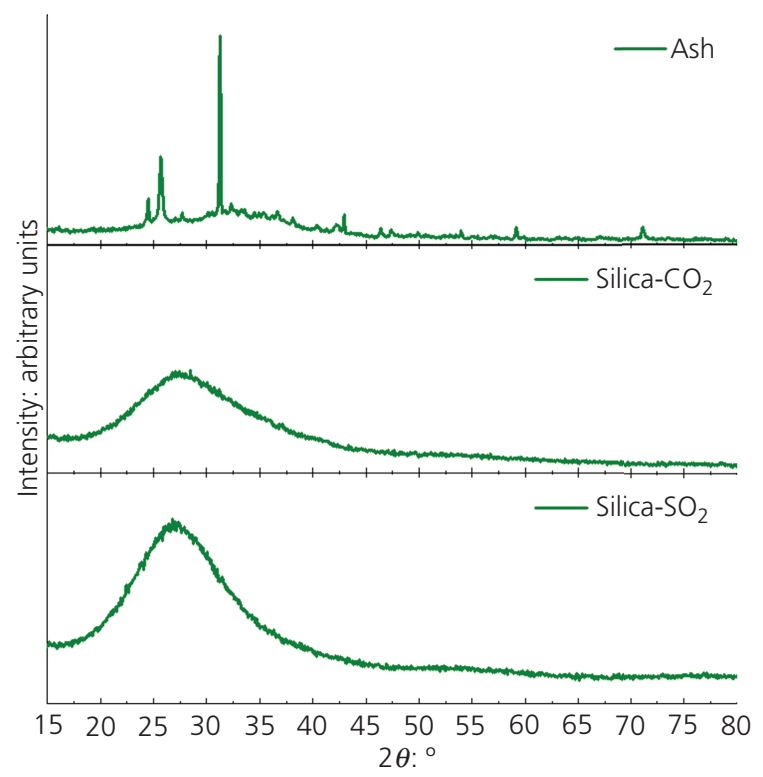

Figure 2. XRD diffraction patterns of ash, silica- $\mathrm{CO}_{2}$ and silica- $\mathrm{SO}_{2}$ 
Table 1. Elemental composition of the precursor and the products obtained by XRF

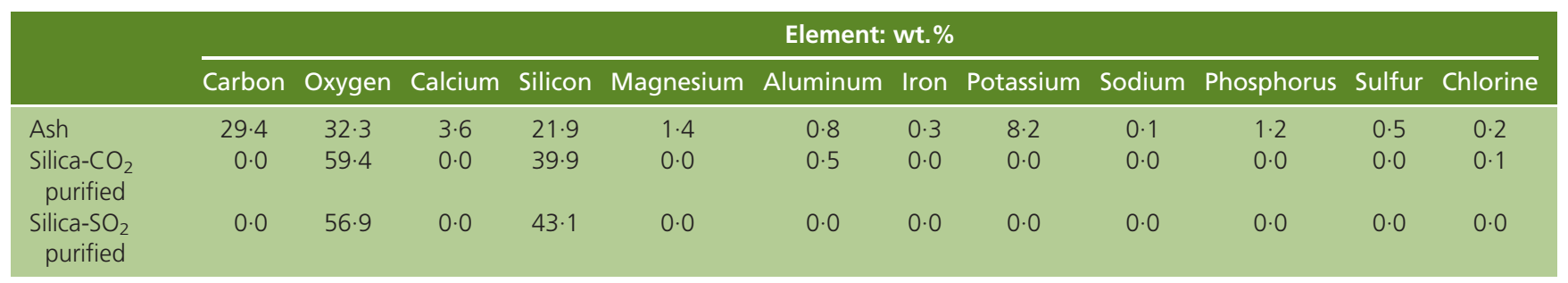

Table 2. Elemental composition of the precursor and the products obtained by EDS

\begin{tabular}{|c|c|c|c|c|c|c|c|c|c|c|c|c|}
\hline & \multicolumn{12}{|c|}{ Element: wt.\% } \\
\hline & Carbon & Oxygen & Calcium & Silicon & Magnesium & Aluminum & Iron & Potassium & Sodium & Phosphorus & Sulfur & Chlorine \\
\hline Ash & $20 \cdot 6$ & $40 \cdot 4$ & $5 \cdot 1$ & $18 \cdot 3$ & $1 \cdot 1$ & 0.6 & 0.6 & $11 \cdot 1$ & $0 \cdot 1$ & 0.9 & 0.7 & 0.5 \\
\hline $\begin{array}{c}\text { Silica- } \mathrm{CO}_{2} \\
\text { purified }\end{array}$ & 0.0 & $53 \cdot 2$ & 0.0 & $46 \cdot 8$ & 0.0 & 0.0 & 0.0 & 0.0 & 0.0 & 0.0 & 0.0 & 0.0 \\
\hline $\begin{array}{c}\text { Silica-SO } \\
\text { purified }\end{array}$ & 0.0 & $54 \cdot 1$ & 0.0 & $55 \cdot 9$ & 0.0 & 0.0 & 0.0 & 0.0 & 0.0 & 0.0 & 0.0 & 0.0 \\
\hline
\end{tabular}

(shown in Table 2); however, the obtained values were slightly different. Note that XRF is a more suitable method for the determination of the net elemental composition, while the information about the composition comes from a relatively large volume. On the other hand, XRF fails to measure accurately light elements (carbon and oxygen); hence, the indirectly determined content of carbon is not very precise.

The sample morphology was studied using SEM. Extremely large particles were found, which are caused by the presence of the amorphous phase sintered together with the crystalline phase (Figure 3). Elemental distribution maps and EDS spectrum are also shown in this figure.

After the thermal treatment of ash with sodium hydroxide, a multiphase product was obtained. The reaction mixture contained a solid agglomerated yellowish phase and a loose black carbon powder phase. According to the XRD of the obtained product, it contained a mixture of phases from which sodium silicate (powder diffraction file (PDF) number 04-010-1394) and sodium carbonate (PDF number 00-055-0503) were dominant. Black carbon was simply swept away and the solid agglomerate was next dissolved in water. The obtained suspension was filtered using a filtration paper. Both the filtrate and the filtration cake were further processed and analyzed. The filtration cake was composed of light and heavy phases. The light phase contained mainly insoluble calcium carbonate, while the heavy phase contained some calcium carbonate, various aluminosilicates and other minor phases. It is expected that during this process, all calcium will be removed, since calcium carbonate or hydroxides are poorly soluble.

The solution then underwent a precipitation using carbon dioxide or sulfur dioxide. The precipitate obtained by carbon dioxide acidification contained not only mostly amorphous silica but also a significant amount of sodium and small amounts of aluminum and potassium. The precipitate from sulfur dioxide contained only a small amount of sodium impurity - this may be attributed to the different solubilities of sodium carbonate and sulfite and also different acidities of the respective gases. Finally, the products of the precipitation were washed by water. After removing soluble components, according to $\mathrm{XRF}$, the silica- $\mathrm{SO}_{2}$ sample contained pure silica, while the silica- $\mathrm{CO}_{2}$ sample contained, in addition to

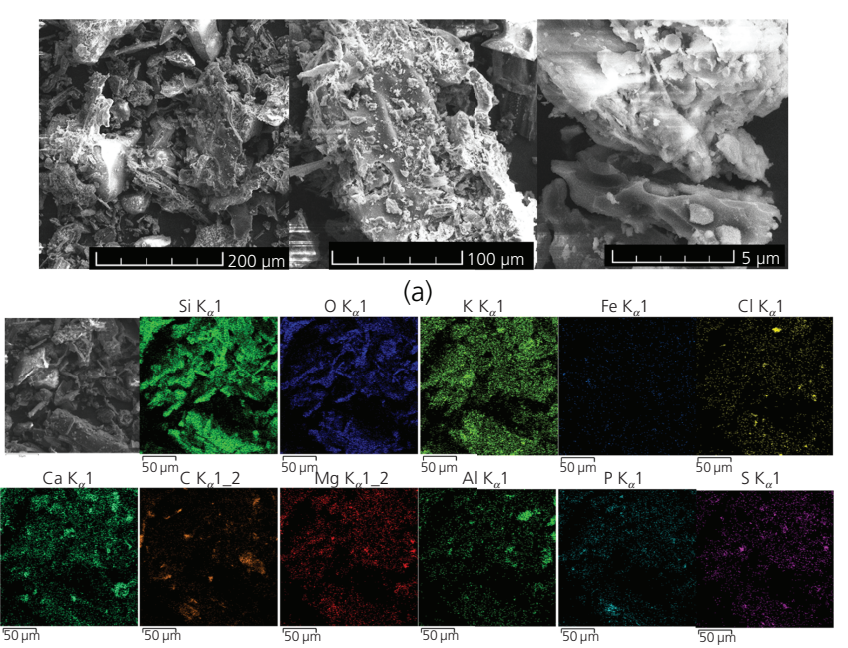

(b)

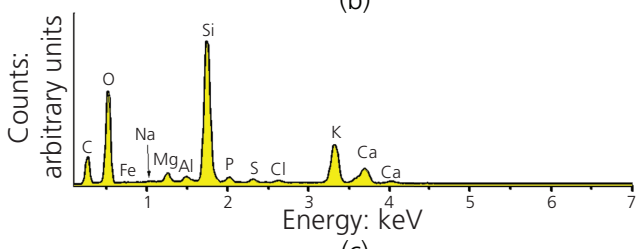

(c)

Figure 3. SEM and EDS analyses of micrographs of wheat straw ash (ash) (a) SEM micrographs, (b) elemental distribution maps and (c) EDS spectrum 

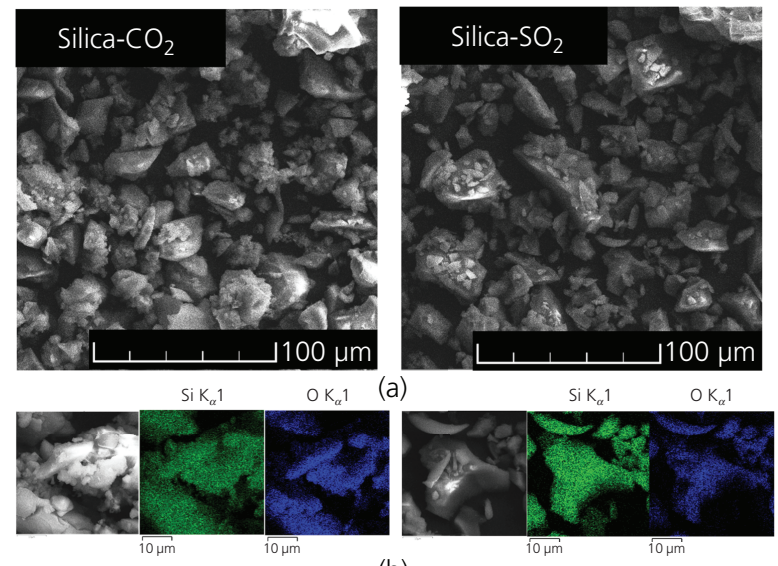

(b)

Figure 4. SEM and EDS analysis of silica- $\mathrm{CO}_{2}$ (left) and silica- $\mathrm{SO}_{2}$ (right). (a) SEM micrographs and (b) elemental distribution maps of silicon and oxygen

the silica, traces of aluminum. This was not confirmed by EDS, indicating only silicon and oxygen in both samples (see Tables 1 and 2 and Figure 4). XRD patterns shown in Figure 2 confirmed a highly amorphous nature of both samples. The morphology was studied using SEM, showing the presence of large agglomerates of amorphous silica (Figure 4). The resulting yields of the obtained amorphous silicon dioxide can be expected to be very high (up to $\sim 80 \%$ ). Some silicon was found in the heavy phase that contained various aluminosilicates, and some silica was lost during the filtration process. However, the process of acidification is quantitative; during the precipitation almost all soluble silicates were transformed into amorphous silica. The precise amount of the respective phases would also depend on the exact composition of the ash, which would vary with the type of biomass ash used.

The light phase obtained during the separation process can also be further utilized in applications where it is desired - for example, in special metallurgy and elsewhere. The phase can be used in the construction industry for various purposes. ${ }^{18}$ In the light phase, calcium is the most abundant element except for oxygen (according to EDS), supporting the hypothesis that insoluble

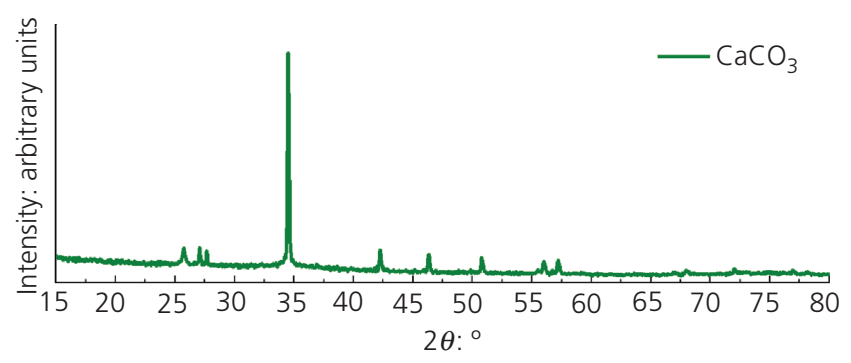

Figure 5. XRD diffraction patterns of the light phase containing calcium carbonate calcium carbonate has been formed during the water treatment of the product. This was confirmed by XRD (see Figure 5).

The carbon dioxide approach can use this greenhouse gas for the acidification of highly alkaline solution, turning it effectively into carbonates. The described approach is mainly interesting in view of the prospective processing of waste material produced industrially in huge quantities; but also of interest is the separation of all components with a potential for further applications. Moreover, the fact that the proposed process is relatively low cost could be important for large-scale utilization.

\section{Conclusions}

The authors demonstrated that wheat straw ash waste can be simply processed in order to obtain pure amorphous silica and other phases that can be further used. The observed results are promising for possible applications - the sulfur dioxide-based process can be used when extra-pure amorphous silica is desired, while the more affordable carbon dioxide process of precipitation may be used in applications not requiring high-purity chemicals. It has to be noted that the regeneration of both gases is also easily possible by simple thickening and subsequent acidification of the waste solution. The process is also promising with respect to its scale-up and implementation in industrial practice because not only is the valuable amorphous silica obtained but the process also provides the possibility of applications of both heavy and light phases. The heavy phase may be used as construction filler, while the light one may be processed in burnt lime facilities.

\section{Acknowledgements}

This work was supported by Czech Science Foundation, Grant Number 17-02815S, and by the Ministry of Education of the Czech Republic, Grant Number 20/2017 for specific university research.

\section{REFERENCES}

1. Ewing CS, Veser G, McCarthy JJ, Lambrecht DS and Johnson JK (2016) Predicting catalyst-support interactions between metal nanoparticles and amorphous silica supports. Surface Science 652: 278-285.

2. Thornburg NE, Nauert SL, Thompson AB and Notestein JM (2016) Synthesis-structure-function relationships of silica-supported niobium (V) catalysts for alkene epoxidation with $\mathrm{H}_{2} \mathrm{O}_{2}$. ACS Catalysis 6(9): 6124-6134.

3. Zabeti M, Baltrusaitis J and Seshan K (2016) Chemical routes to hydrocarbons from pyrolysis of lignocellulose using Cs promoted amorphous silica alumina catalyst. Catalysis Today 269: 156-165.

4. Bulut MO, Cimen O, Akbulut Y, Akcali K and Dereli B (2015) Application of amorphous silica, colemanite and pumice on cotton fabric by screen printing method. Industria Textila 66(5): 289-296.

5. Verraedt E, Van den Mooter G and Martens JA (2011) Novel amorphous microporous silica spheres for controlled release applications. Journal of Pharmaceutical Sciences 100(10): 4295-4301.

6. Aerts CA, Verraedt E, Mellaerts R et al. (2007) Tunability of pore diameter and particle size of amorphous microporous silica for diffusive controlled release of drug compounds. Journal of Physical Chemistry C 111(36): 13404-13409.

7. Nodera A and Kanai T (2006) Flame retardancy of polycarbonate-polydimethylsiloxane block copolymer/silica nanocomposites. Journal of Applied Polymer Science 101(6): $3862-3868$. 
Green Materials

Volume 6 Issue GMAT1
Production of pure amorphous silica from wheat straw ash

Bartůněk, Sedmidubský, Bouša and Jankovský
8. Hou XF, Ding H, Zheng YX and Wang MM (2013) Preparation and characterisation of amorphous silica/anatase composite through mechanochemical method. Materials Research Innovations 17: S234-S239.

9. Maslova MV, Gerasimova LG and Okhrimenko RF (2011) Functional role of amorphous silica in the composite based on titanium phosphate ion exchanger. Glass Physics and Chemistry 37(1): 65-71.

10. Xiao ZQ, Tan FT, Wang W et al. (2014) Oxidation protection of Ti6Al-4V alloy using a novel glass-amorphous silica composite coating. Ceramics International 40(2): 3503-3509.

11. Zemnukhova LA and Panasenko AE (2013) A novel composite material based on antimony(III) oxide and amorphous silica. Journal of Solid State Chemistry 201: 9-12.

12. Liu DD, Fang L and Cheng FQ (2016) Bisurfactant-assisted preparation of amorphous silica from fly ash. Asia-Pacific Journal of Chemical Engineering 11(6): 884-892.

13. Shim J, Velmurugan P and Oh BT (2015) Extraction and physical characterization of amorphous silica made from corn cob ash at variable $\mathrm{pH}$ conditions via sol gel processing. Journal of Industrial and Engineering Chemistry 30: 249-253.

14. Khorsand $\mathrm{H}$, Kiayee $\mathrm{N}$ and Masoomparast $\mathrm{AH}$ (2013) Optimization of amorphous silica nanoparticles synthesis from rice straw ash using design of experiments technique. Particulate Science and Technology 31(4): 366-371.

15. Thompson JC (1989) Dangers of hydrofluoric acid. American Journal of Physics 57(3): 200-201.

16. El Bouseily AM and Elshamy TM (1975) Wet preparation of 'water glass' from hull ash of Egyptian rice. Nature 256(5514): 198-199.

17. Sander ML and Andren O (1997) Ash from cereal and rape straw used for heat production: liming effect and contents of plant nutrients and heavy metals. Water Air and Soil Pollution 93(1-4): 93-108.

18. Zaleska M, Pavlik Z, Pavlikova M et al. (2018) Biomass ash-based mineral admixture prepared from municipal sewage sludge and its application in cement composites. Clean Technologies and Environmental Policy 20(1): 159-171.

\section{How can you contribute?}

To discuss this paper, please submit up to 500 words to the journal office at journals@ice.org.uk. Your contribution will be forwarded to the author(s) for a reply and, if considered appropriate by the editor-in-chief, it will be published as a discussion in a future issue of the journal.

ICE Science journals rely entirely on contributions from the field of materials science and engineering. Information about how to submit your paper online is available at www.icevirtuallibrary.com/page/authors, where you will also find detailed author guidelines. 